International Journal of Pure and Applied Mathematics

Volume 95 No. 1 2014, 69-78

ISSN: 1311-8080 (printed version); ISSN: 1314-3395 (on-line version)

url: http://www.ijpam.eu

doi: http://dx.doi.org/10.12732/ijpam.v95i1.8

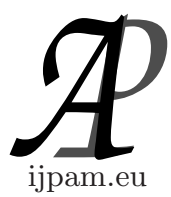

\title{
STABILITY ANALYSIS OF SWITCHED DISCRETE TIME-DELAY SYSTEMS WITH CONVEX POLYTOPIC UNCERTAINTIES
}

\author{
Manlika Rajchakit ${ }^{1}$, Grienggrai Rajchakit ${ }^{2} \S$ \\ ${ }^{1,2}$ Department of Mathematics and Statistics \\ Maejo University \\ Chiangmai, 50290, THAILAND
}

\begin{abstract}
This article is concerned with robust stability of switched discrete time-delay systems with convex polytopic uncertainties. The system to be considered is subject to interval time-varying delays, which allows the delay to be a fast time-varying function and the lower bound is not restricted to zero. Based on the discrete Lyapunov functional, a switching rule for robust stability for switched system with convex polytopic uncertainties is designed via linear matrix inequalities.
\end{abstract}

AMS Subject Classification: 47N10, 93C55, 93D20, 94C10

Key Words: switching design, convex polytopic uncertainties, robust stability, interval time-varying delays, Lyapunov function, linear matrix inequalities

\section{Introduction}

Many dynamical systems in real world involve variables that be always confined to the positive orthant, and such systems are generally termed as positive systems in the literature. In particular, positive linear systems have drawn considerable interest due to their numerous applications in the areas such as

Received: January 30, 2014

(C) 2014 Academic Publications, Ltd.

${ }^{\S}$ Correspondence author url: www.acadpubl.eu 
economics, biology, communications, etc. In the context of positive linear systems, stability analysis is a major concern and the so-called linear copositive Lyapunov function has been proved to be an efficient approach. Also, based on the linear copositive Lyapunov function approach, the analysis and synthesis problems of positive linear systems with time delay have been successfully tackled.

A switched system with time-delay individual subsystems is called a switched time-delay system; in particular, when the subsystems are linear, it is then called a switched time-delay linear system. By using the Floquet theory, the author gives new sufficient conditions for stabilize in terms of a controllability rank condition for a linear time-invariant discrete system as well as of matrix inequalities in [1-8]. During the last decades, the stability analysis of switched linear continuous/discrete time-delay systems has attracted a lot of attention $[4,9,10,14]$. The main approach for stability analysis relies on the use of Lyapunov-Krasovskii functional and linear matrix inequality (LMI) approach for constructing a common Lyapunov function [11-18]. Although many important results have been obtained for switched linear continuous-time systems, there are a few results concerning the stability of switched linear discrete systems with time-varying delays. It was shown in [11-17] that when all subsystems are asymptotically stable, the switching system is asymptotically stable under an arbitrary switching rule. The asymptotic stability for switching linear discrete time-delay systems has been studied in [19], but the result was limited to constant delays. In [20], a class of switching signals has been identified for the considered switched discrete-time delay systems to be stable under the average dwell time scheme.

This paper studies the robust stability problem for switched linear discrete systems with convex polytopic uncertainties with interval time-varying delays. Specifically, our goal is to develop a constructive way to design a switching rule to be robustly stable the system. By using improved Lyapunov-Krasovskii functional combined with the LMIs technique, we propose new criteria for the robust stability of the system. Compared to the existing results, our result has its own advantages. First, the time delay is assumed to be a time-varying function belonging to a given interval, which means that the lower and upper bounds for the time-varying delay are available, the delay function is bounded but not restricted to zero. Second, the approach allows us to design the switching rule for robust stability in terms of LMIs, which can be solvable by utilizing Matlab's LMI Control Toolbox available in the literature to date.

The paper is organized as follows: Section 2 presents definitions and some well-known technical propositions needed for the proof of the main results. The 
robust stability of switched discrete time-delay systems with convex polytopic uncertainties is presented in Section 3.

\section{Preliminaries}

The following notations will be used throughout this paper. $R^{+}$denotes the set of all real non-negative numbers; $R^{n}$ denotes the $n$-dimensional space with the scalar product of two vectors $\langle x, y\rangle$ or $x^{T} y ; R^{n \times r}$ denotes the space of all matrices of $(n \times r)$ - dimension. $A^{T}$ denotes the transpose of $A$; a matrix $A$ is symmetric if $A=A^{T}$.

Matrix $A$ is semi-positive definite $(A \geq 0)$ if $\langle A x, x\rangle \geq 0$, for all $x \in R^{n} ; A$ is positive definite $(A>0)$ if $\langle A x, x\rangle>0$ for all $x \neq 0 ; A \geq B$ means $A-B \geq 0$. $\lambda(A)$ denotes the set of all eigenvalues of $A ; \lambda_{\min }(A)=\min \{\operatorname{Re} \lambda: \lambda \in \lambda(A)\}$.

Consider a discrete systems with convex polytopic uncertainties with interval time-varying delay of the form

$$
\begin{aligned}
x(k+1) & =A(\zeta)_{\gamma} x(k)+B(\zeta)_{\gamma} x(k-d(k)), \quad k=0,1,2, \ldots \\
x(k) & =v_{k}, \quad k=-d_{2},-d_{2}+1, \ldots, 0,
\end{aligned}
$$

where $x(k) \in R^{n}$ is the state, $\gamma():. R^{n} \rightarrow \mathcal{N}:=\{1,2, \ldots, N\}$ is the switching rule, which is a function depending on the state at each time and will be designed. A switching function is a rule which determines a switching sequence for a given switching system. Moreover, $\gamma(x(k))=i$ implies that the system realization is chosen as the $i^{\text {th }}$ system, $i=1,2, \ldots, N$. It is seen that the system (1) can be viewed as an autonomous switched system in which the effective subsystem changes when the state $x(k)$ hits predefined boundaries. $A_{i}, B_{i}, i=1,2, \ldots, N$ are given constant matrices. The system matrices are subjected to uncertainties and belong to the polytopes $\Omega$ given by

$$
\Omega=\left\{\left[A_{i j}, B_{i j}\right](\zeta):=\sum_{j=1}^{N} \zeta_{j}\left[A_{i j}, B_{i j}\right], \quad \sum_{j=1}^{N} \zeta_{j}=1, \zeta_{j} \geq 0\right\}
$$

where $A_{i j}, B_{i j}, i, j=1,2, \ldots, N$, are given constant matrices with appropriate dimensions. The time-varying function $d(k)$ satisfies the following condition:

$$
0<d_{1} \leq d(k) \leq d_{2}, \quad \forall k=0,1,2, \ldots
$$


Remark 1. It is worth noting that the time delay is a time-varying function belonging to a given interval, in which the lower bound of delay is not restricted to zero.

Definition 1. The switched linear discrete systems with convex polytopic uncertainties (1) is robustly stable if there exist a positive definite scalar function $V\left(k, x(k): R^{+} \times R^{n} \rightarrow R\right.$ and a switching function $\gamma($.$) such that$

$$
\Delta V(k, x(k))=V(k+1, x(k+1))-V(k, x(k))<0,
$$

along any trajectory of solution of the system (1) for all uncertainties which satisfy $(2)$.

Definition 2. The switched system (1) is robustly stable if there exists a switching function $\gamma($.$) such that the zero solution of the system is robustly$ stable.

Definition 3. The system of matrices $\left\{J_{i}\right\}, i=1,2, \ldots, N$, is said to be strictly complete if for every $x \in R^{n} \backslash\{0\}$ there is $i \in\{1,2, \ldots, N\}$ such that $x^{T} J_{i} x<0$.

It is easy to see that the system $\left\{J_{i}\right\}$ is strictly complete if and only if

$$
\bigcup_{i=1}^{N} \alpha_{i}=R^{n} \backslash\{0\}
$$

where

$$
\alpha_{i}=\left\{x \in R^{n}: \quad x^{T} J_{i} x<0\right\}, i=1,2, \ldots, N .
$$

Proposition 1. (see [21]) The system $\left\{J_{i}\right\}, i=1,2, \ldots, N$, is strictly complete if there exist $\delta_{i} \geq 0, i=1,2, \ldots, N, \sum_{i=1}^{N} \delta_{i}>0$ such that

$$
\sum_{i=1}^{N} \delta_{i} J_{i}<0
$$

If $N=2$ then the above condition is also necessary for the strict completeness.

Proposition 2. For real numbers $\zeta_{j} \geq 0, j=1,2, \ldots, N, \sum_{j=1}^{N} \zeta_{j}=1$, the following inequality hold

$$
(N-1) \sum_{j=1}^{N} \zeta_{j}^{2}-2 \sum_{j=1}^{N-1} \sum_{l=j+1}^{N} \zeta_{j} \zeta_{l} \geq 0 .
$$


Proof. The proof follows by completing the square:

$$
(N-1) \sum_{j=1}^{N} \zeta_{j}^{2}-2 \sum_{j=1}^{N-1} \sum_{l=j+1}^{N} \zeta_{j} \zeta_{l}=\sum_{j=1}^{N-1} \sum_{l=j+1}^{N}\left(\zeta_{j}-\zeta_{l}\right)^{2} \geq 0 .
$$

\section{Main Results}

Let us set

$$
\begin{aligned}
& W_{i j j}(P, Q, R) \\
& =\left(\begin{array}{ccc}
\left(d_{2}-d_{1}+1\right) Q_{j}-P_{j}-A_{i j}^{T} R_{j}-R_{j}^{T} A_{i j} & R_{j}^{T}-A_{i j}^{T} R_{j} & -R_{j}^{T} B_{i j} \\
R_{j}-R_{j}^{T} A_{i j} & P_{j}+R_{j}+R_{j}^{T} & -R_{j}^{T} B_{i j} \\
-B_{i j}^{T} R_{j} & -B_{i j}^{T} R_{j} & -Q_{j}
\end{array}\right)^{\prime} \\
& W_{i j l}(P, Q, R) \\
& =\left(\begin{array}{ccc}
\left(d_{2}-d_{1}+1\right) Q_{j}-P_{j}-A_{i l}^{T} R_{j}-R_{j}^{T} A_{i l} & R_{j}^{T}-A_{i l}^{T} R_{j} & -R_{j}^{T} B_{i l} \\
R_{j}-R_{j}^{T} A_{i l} & P_{j}+R_{j}+R_{j}^{T} & -R_{j}^{T} B_{i l} \\
-B_{i l}^{T} R_{j} & -B_{i l}^{T} R_{j} & -Q_{j}
\end{array}\right), \\
& W_{i l j}(P, Q, R) \\
& =\left(\begin{array}{ccc}
\left(d_{2}-d_{1}+1\right) Q_{l}-P_{l}-A_{i j}^{T} R_{l}-R_{l}^{T} A_{i j} & R_{l}^{T}-A_{i j}^{T} R_{l} & -R_{l}^{T} B_{i j} \\
R_{l}-R_{l}^{T} A_{i j} & P_{l}+R_{l}+R_{l}^{T} & -R_{l}^{T} B_{i j} \\
-B_{i j}^{T} R_{l} & -B_{i j}^{T} R_{l} & -Q_{l}
\end{array}\right)^{2} \\
& \mathcal{R}=\left(\begin{array}{lll}
R & 0 & 0 \\
0 & 0 & 0 \\
0 & 0 & 0
\end{array}\right), P(\zeta)=\sum_{j=1}^{N} \zeta_{j} P_{j}, Q(\zeta)=\sum_{j=1}^{N} \zeta_{j} Q_{j}, R(\zeta)=\sum_{j=1}^{N} \zeta_{j} R_{j}, \\
& \lambda_{1}=\lambda_{\min }(P) \text {, }
\end{aligned}
$$

Theorem 1. The switched system with convex polytopic uncertainties (1) is robustly stable if there exist symmetric matrices $P_{i}>0, Q_{i}>0, \mathcal{R} \geq 0, i=$ $1,2 \ldots, N$ and matrix $R_{i}, i=1,2 \ldots, N$ satisfying the following conditions

$$
\begin{aligned}
& (i) W_{i j j}(P, Q, R)+\mathcal{R}<0, i=1,2, \ldots, N, j=1,2, \ldots, N . \\
& (i i) W_{i j l}(P, Q, R)+W_{i l j}(P, Q, R)-\frac{2}{N-1} \mathcal{R}<0, i=1,2, \ldots, N, \\
& j=1,2, \ldots, N-1 ; l=j+1, \ldots, N .
\end{aligned}
$$

The switching rule is chosen as $\gamma(x(k))=i$. 
Proof. Consider the following Lyapunov-Krasovskii functional for any $i$ th system (1)

$$
V(k)=V_{1}(k)+V_{2}(k)+V_{3}(k),
$$

where

$$
\begin{gathered}
V_{1}(k)=x^{T}(k) P(\zeta) x(k), \quad V_{2}(k)=\sum_{i=k-d(k)}^{k-1} x^{T}(i) Q(\zeta) x(i), \\
V_{3}(k)=\sum_{j=-d_{2}+2}^{-d_{1}+1} \sum_{l=k+j+1}^{k-1} x^{T}(l) Q(\zeta) x(l),
\end{gathered}
$$

We can verify that

$$
\lambda_{1}\|x(k)\|^{2} \leq V(k) .
$$

Let us set $\xi(k)=[x(k) x(k+1) x(k-d(k))]^{T}$, and

$$
H=\left(\begin{array}{ccc}
0 & 0 & 0 \\
0 & P(\zeta) & 0 \\
0 & 0 & 0
\end{array}\right), \quad G=\left(\begin{array}{ccc}
P(\zeta) & 0 & 0 \\
R(\zeta) & R(\zeta) & 0 \\
0 & 0 & I
\end{array}\right)
$$

Then, the difference of $V_{1}(k)$ along the solution of the system is given by

$$
\begin{aligned}
\Delta V_{1}(k) & =x^{T}(k+1) P(\zeta) x(k+1)-x^{T}(k) P(\zeta) x(k) \\
& =\xi^{T}(k) H(\zeta) \xi(k)-2 \xi^{T}(k) G^{T}(\zeta)\left(\begin{array}{c}
0.5 x(k) \\
0 \\
0
\end{array}\right)
\end{aligned}
$$

Therefore, from (6) it follows that

$$
\Delta V_{1}(k)=\xi^{T}(k) W_{i}(P(\zeta), Q(\zeta), R(\zeta)) \xi(k)
$$

where

$$
\begin{aligned}
W_{i}(P(\zeta), Q(\zeta), R(\zeta)) & =\left(\begin{array}{ccc}
0 & 0 & 0 \\
0 & P(\zeta) & 0 \\
0 & 0 & 0
\end{array}\right)-G^{T}(\zeta)\left(\begin{array}{ccc}
0.5 I & 0 & 0 \\
A_{i}(\zeta) & -I & B_{i}(\zeta) \\
0 & 0 & 0
\end{array}\right) \\
& -\left(\begin{array}{ccc}
0.5 I & A_{i}^{T}(\zeta) & 0 \\
0 & -I & 0 \\
0 & B_{i}^{T}(\zeta) & 0
\end{array}\right) G(\zeta) .
\end{aligned}
$$


The difference of $V_{2}(k)$ is given by

$$
\begin{aligned}
& \Delta V_{2}(k) \\
& \leq \sum_{i=k+1-d(k+1)}^{k-d_{1}} x^{T}(i) Q(\zeta) x(i)+x^{T}(k) Q(\zeta) x(k)-x^{T}(k-d(k)) Q(\zeta) x(k-d(k)) .
\end{aligned}
$$

The difference of $V_{3}(k)$ is given by

$$
\Delta V_{3}(k)=\left(d_{2}-d_{1}\right) x^{T}(k) Q(\zeta) x(k)-\sum_{j=k+1-d_{2}}^{k-d_{1}} x^{T}(j) Q(\zeta) x(j) .
$$

We obtain from (9) and (10) that

$$
\begin{aligned}
& \Delta V_{2}(k)+\Delta V_{3}(k) \\
& \leq\left(d_{2}-d_{1}+1\right) x^{T}(k) Q(\zeta) x(k)-x^{T}(k-d(k)) Q(\zeta) x(k-d(k)) .
\end{aligned}
$$

Therefore, combining the inequalities (7), (11) gives

$$
\Delta V(k) \leq \xi^{T}(k) W_{i}(P(\zeta), Q(\zeta), R(\zeta)) \xi(k),
$$

where

$$
\psi(k)=[x(k) x(k+1) x(k-d(k))]^{T} .
$$

Therefore, we finally obtain from (12) and the condition (i), (ii) that

$$
\Delta V(k)<\xi^{T}(k) W_{i}(P(\zeta), Q(\zeta), R(\zeta)) \xi(k)<0, \quad \forall i=1,2, \ldots, N, k=0,1,2, \ldots
$$

which, combining the condition (5) and the Lyapunov stability theorem [21], concludes the proof of the theorem.

Remark 2. Note that the result is proposed in [3-9] for switching systems to be asymptotically stable under an arbitrary switching rule. The asymptotic stability for switching linear discrete time-delay systems studied in [14-19] was limited to constant delays. In [18-20], a class of switching signals has been identified for the considered switched discrete-time delay systems to be stable under the averaged well time scheme. 


\section{Conclusion}

This paper has proposed a switching design for robust stability of switched discrete time-delay systems with convex polytopic uncertainties with interval time-varying delays. Based on the discrete Lyapunov functional, a switching rule for the robust stability of switched discrete time-delay systems with convex polytopic uncertainties is designed via linear matrix inequalities.

\section{Acknowledgements}

This work was supported by the Thailand Research Fund Grant, the Commission for Higher Education and Faculty of Science, Maejo University, Thailand. The authors thank anonymous reviewers for valuable comments and suggestions, which allowed us to improve the paper.

\section{References}

[1] D. Liberzon and A.S. Morse, Basic problems in stability and design of switched systems, IEEE Control Syst. Mag. 19 (1999) 57-70.

[2] A. V. Savkin and R. J. Evans, Hybrid Dynamical Systems: Controller and Sensor Switching Problems, Springer, New York, 2001.

[3] K. Ratchagit, Asymptotic stability of nonlinear delay-difference system via matrix inequalities and application, International Journal of Computational Methods, pp. 389-397, 2009. DOI: 10.1142/S0219876209001899

[4] Manlika Rajchakit and Grienggrai Rajchakit, Mean Square Exponential Stability of Stochastic Switched System with Interval Time-Varying Delays, Abstract and Applied Analysis, vol. 2012, Article ID 623014, 12 pages, 2012. doi: $10.1155 / 2012 / 623014$

[5] C. H. Lien, K. W. Yu, Y. J. Chung, Y. F. Lin, L. Y. Chung and J. D. Chen, Exponential stability analysis for uncertain switched neutral systems with interval-time-varying state delay, Nonlinear Analysis: Hybrid systems $\mathbf{3}$ (2009) 334-342.

[6] K. Ratchagit and V. N. Phat, Robust Stability and Stabilization of Linear Polytopic Delay-Difference Equations with Interval Time-Varying Delays, Neural, Parallel, and Scientific Computations, 19(2011), 361-372. 
[7] V. N. Phat and K. Ratchagit, Stability and stabilization of switched linear discrete-time systems with interval time-varying delay, Nonlinear Analysis: Hybrid Systems, 5(2011), 605-612. DOI: 10.1016/j.nahs.2011.05.006

[8] S.Xu and J. Lam, Robust stability and stabilization of discrete singular systems: An equivalent characterization, IEEE Trans. Automat. Contr., 49(2004), 568-574.

[9] S.P. Ma, Z.L. Cheng, An LMI approach to robust stabilization for uncertain discrete-time singular systems, In Proceedings of the 41st IEEE CDC, Las Vegas, Nevada, USA, 2002(2002), 1090-1095.

[10] Kreangkri Ratchagit, STABILITY ANALYSIS OF LINEAR SYSTEMS WITH TIME DELAYS, International Journal of Pure and Applied Mathematics, Vol. 76 No. 1, 2012, 21-28.

[11] K. Ratchagit, THE SUFFICIENT CONDITIONS FOR STABILITY OF LINEAR TIME-VARYING SYSTEMS WITH STATE DELAYS, International Journal of Pure and Applied Mathematics, Vol. 65 No. 1, 2010, $65-72$.

[12] Kreangkri Ratchagit, STABILITY OF LINEAR TIME-VARYING SYSTEMS, International Journal of Pure and Applied Mathematics, Vol. 63, No. 4, 2010, 411-417.

[13] P. Shi, E.K. Boukas, On H1 control design for singular continuoustime delay systems with parametric uncertainties, Nonlinear Dynamics and Systems Theory, 4(2004), 59-71.

[14] K. Ratchagit and V.N. Phat, Stability criterion for discrete-time systems, J. Ineq. Appl., 2010(2010), 1-6. doi:10.1155/2010/201459

[15] Grienggrai Rajchakit, Delay-Dependent Asymptotical Stabilization Criterion of Recurrent Neural Networks, Applied Mechanics and Materials. 330(2013) 1045-1048. doi:10.4028/www.scientific.net/AMM.330.1045

[16] G. Xie and L. Wang, Quadratic stability and stabilization of discretetime switched systems with state delay, In: Proc. of the IEEE Conference on Decision and Control, Atlantics, December 2004, 3235-3240.

[17] S. Boyd, L. E. Ghaoui, E. Feron and V. Balakrishnan, Linear Matrix Inequalities in System and Control Theory, SIAM, Philadelphia, 1994. 
[18] K. Ratchagit, A switching rule for the asymptotic stability of discrete-time systems with convex polytopic uncertainties, Asian-European Journal of Mathematics, Vol. 5, 2012. DOI: 10.1142/S1793557112500258

[19] G. Rajchakit, Stabilization of switched discrete-time systems with convex polytopic uncertainties, Journal of Computational Analysis and Applications, Vol. 16, 2014, 20-29.

[20] W. A. Zhang and Li Yu, Stability analysis for discrete-time switched timedelay systems, Automatica 45 (2009) 2265-2271.

[21] R. P. Agarwal, Difference Equations and Inequalities, Second Edition, Marcel Dekker, New York, 2000. 